\title{
Síntesis óptima de un mecanismo plano para seguimiento de trayectoria utilizando evolución diferencial
}

\author{
Eduardo Vega-Alvarado ${ }^{1}$, Eric Santiago-Valentín ${ }^{1}$, Alvaro Sánchez-Márquez ${ }^{2}$, \\ Adrián Solano-Palma ${ }^{1}$, Edgar Alfredo Portilla-Flores ${ }^{1}$, Leticia Flores-Pulido ${ }^{2}$ \\ ${ }^{1}$ Instituto Politécnico Nacional, \\ Centro de Innovación y Desarrollo Tecnológico en Cómputo, \\ México D.F., México \\ ${ }^{2}$ Universidad Autónoma de Tlaxcala, \\ Facultad de Ciencias Básicas, Ingeniería y Tecnología, \\ Apizaco, Tlaxcala, México \\ \{evega, asolanop, aportilla\}@ipn.mx, asanchez@uat.mx, \\ \{e.santiago.valentin, aicitel.flores\}@gmail.com
}

\begin{abstract}
Resumen. En este trabajo se presenta la síntesis de un mecanismo de cuatro barras para seguimiento de una trayectoria lineal de seis puntos. Para resolver el problema de optimización numérica con restricciones asociado al mecanismo se utiliza el algoritmo de evolución diferencial, modificado para aplicar una selección tipo torneo basada en las reglas de factibilidad de Deb. Los resultados alcanzados muestran una muy alta precisión en el seguimiento de la trayectoria propuesta, superando los valores mínimos obtenidos en otros trabajos similares.
\end{abstract}

Palabras clave: Síntesis, optimización, mecanismo de cuatro barras, evolución diferencial, restricciones.

\section{Introducción}

Uno de los mecanismos que más se utiliza en el diseño de maquinaria es el de cuatro barras, debido a que se ha comprobado que en sus diferentes configuraciones es el mecanismo articulado más simple para movimiento controlado de un grado de libertad [1].

Sin pérdida de generalidad, en el contexto de ingeniería mecánica el término de síntesis se entiende como el proceso de diseño de una máquina o sistema mecánico [2]. Existen diferentes tipos de síntesis, en el presente trabajo se abordará la síntesis dimensional.

La síntesis dimensional de un mecanismo es la determinación de los tamaños (longitudes) de los eslabones necesarios para realizar los movimientos deseados[1]. En este sentido, es importante determinar la tarea a realizar por el mecanismo para determinar que tipo de síntesis dimensional se llevará a cabo: 
generación de función, trayectoria o movimiento. En la síntesis para generación de función, se realiza la correlación de un movimiento de entrada con un movimiento de salida en un mecanismo; en lo que respecta a la generación de trayectoria se define como el control de un punto en el plano tal que siga alguna trayectoria prescrita y finalmente, la generación de movimiento se define como el control de una línea en el plano, tal que asume algún conjunto prescrito de posiciones secuenciales [1].

La forma matemática para expresar un problema de optimización es:

$$
\text { minimizar/maximizar } f(\boldsymbol{x})
$$

sujeto a las restricciones:

$$
\begin{aligned}
& g_{j}(\boldsymbol{x}) \leq 0, \quad j=1,2, \ldots, p \\
& h_{j}(\boldsymbol{x})=0, \quad j=1,2, \ldots, q
\end{aligned}
$$

donde $\boldsymbol{x}$ es el vector de variables de dimensión $n, f(\boldsymbol{x})$ es la función objetivo, $g_{j}(\boldsymbol{x})$ es el conjunto de $p$ restricciones de desigualdad y finalmente $h_{j}(\boldsymbol{x})$ es el conjunto de $q$ restricciones de igualdad.

El diseño de un mecanismo de cuatro barras que siga una trayectoria rectilínea es un caso típico de optimización dura. Los problemas duros de optimización son aquellos que no pueden resolverse de manera óptima o hasta un límite garantizado por medio de métodos determinísticos en un tiempo aceptable. Las metaheurísticas son algoritmos diseñados para resolver de manera aproximada un rango amplio de problemas duros de optimización. En general, las metaheurísticas tienen las siguientes características: están inspiradas en la naturaleza, hacen uso de componentes estocásticos (involucrando variables aleatorias), y tienen una serie de parámetros que requieren ser ajustados al problema a resolver [3].

Para la síntesis de mecanismos seguidores de cuatro barras se han utilizado diversas metaheurísticas en forma de algoritmos evolutivos. En [4], Cabrera et al. aplican el Algoritmo Genético (GA) con modificaciones para el manejo de restricciones, mientras que Bulatović y Dordević [5] emplean Evolución Diferencial (DE) y un método de control variable de desviaciones. En [6], Acharyya y Mandal presentan un comparativo de resultados entre tres algoritmos evolutivos diferentes para la solución del problema(Algoritmo Genético, Evolución Diferencial y Optimización por Cúmulo de Partículas (PSO)), en tanto que Matekar et al. [7] utilizan Evolución Diferencial y una función modificada de error.

En el presente trabajo se utiliza el algoritmo de Evolución Diferencial para la síntesis óptima de un mecanismo de cuatro barras seguidor de trayectoria, el cual es una versión modificada que incluye las reglas de factibilidad de Deb [8] en la etapa de competencia entre la población.La DE es un método usado ampliamente en problemas de optimización global, debido a su rápida convergencia y facilidad de implementación [9]. Por su parte, los criterios de Deb permiten mejorar la selección entre las generaciones, eligiendo al individuo más factible, 
Síntesis óptima de un mecanismo plano para seguimiento de trayectoria utilizando evolución ...

y sustituyendo así al método convencional de escoger al individuo con el mejor valor de la función objetivo.

Este artículo está organizado de la siguiente forma: la Sección 2 describe el problema de síntesis de mecanismos, con una breve explicación de la cinemática tanto del mecanismo como de su acoplador. En la Sección 3 se presentan las estrategias de optimización, analizando a la función objetivo y a las restricciones de diseño. Posteriormente, en la Sección 4 se trata el diseño óptimo del mecanismo, detallando las variables de diseño e introduciendo el problema de optimización. En la Sección 5 se muestra el algoritmo empleado, enfatizando las partes modificadas con respecto a la evolución diferencial tradicional y algunos aspectos de la implementación computacional. Finalmente, en la Sección 6 se presentan y discuten los resultados obtenidos, y en la Sección 7 se exponen las conclusiones correspondientes.

\section{Problema de síntesis del mecanismo}

Sea el mecanismo de cuatro barras que se muestra en la Figura 1, integrado de los siguientes elementos: barra de referencia $\left(r_{1}\right)$, barra de entrada o manivela $\left(r_{2}\right)$, biela o acoplador $\left(r_{3}\right)$ y barra de salida o balancín $\left(r_{4}\right)$.

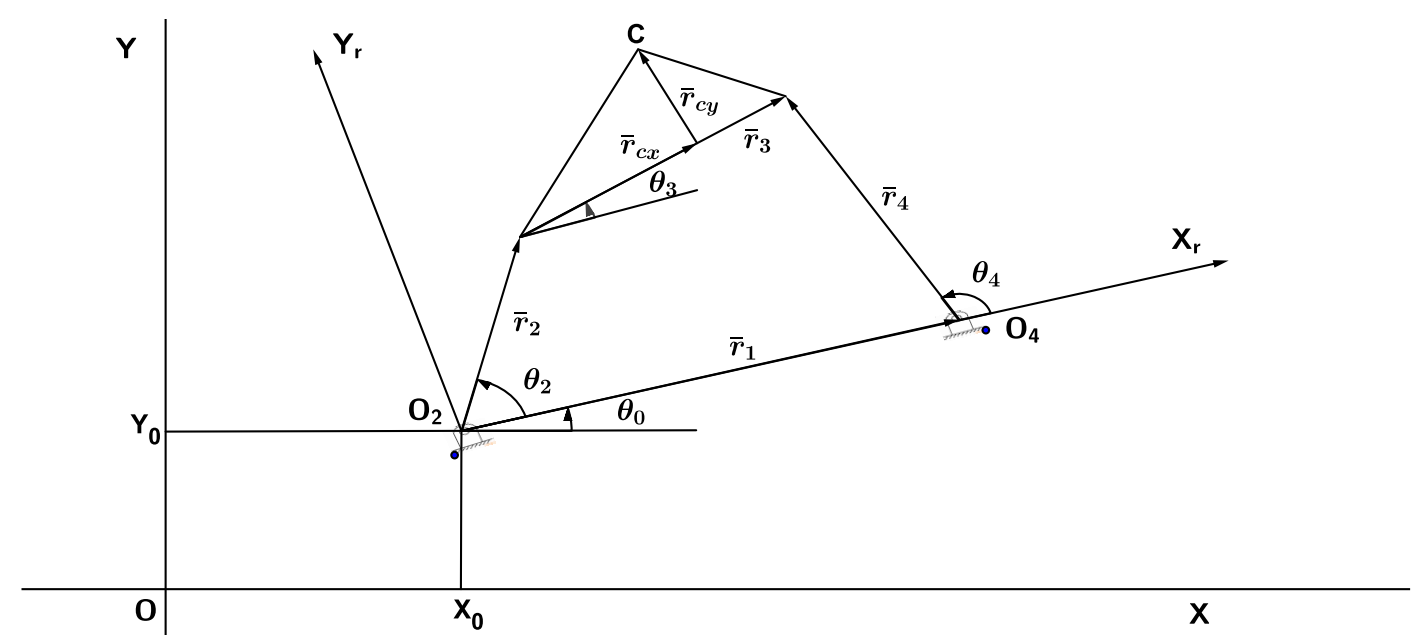

Fig. 1. Mecanismo de cuatro barras.

Con el propósito de establecer la cinemática del mecanismo se proponen dos sistemas coordenados: el fijo al mundo real denominado $0 X Y$ y el de referencia denominado $0 X_{r} Y_{r}$; donde $\left(x_{0}, y_{0}\right)$ es la distancia entre los orígenes de ambos sistemas coordenados, $\theta_{0}$ es el ángulo de rotación del sistema de referencia y $\theta_{i}$ en el que $i=1,2,3,4$ corresponde a los ángulos de las barras del mecanismo; 
finalmente, el punto $C$ del acoplador se determina mediante las coordenadas $\left(r_{c x}, r_{c y}\right)$.

El problema que se resuelve en el presente trabajo es el de la generación de trayectoria para un conjunto de posiciones o puntos de precisión del acoplador sin sincronización prescrita. Esto es, el punto $C$ del acoplador debe tocar un número $N$ de puntos en forma consecutiva sin una secuencia establecida en la barra de entrada para alcanzar dichas posiciones.

\subsection{Cinemática del mecanismo}

La cinemática del mecanismo de cuatro barras ha sido extensamente estudiada, una explicación detallada de la misma se puede consultar en [10]. Para el presente trabajo se considera el análisis de posición del mecanismo.

Del mecanismo propuesto se puede establecer la ecuación de cierre de circuito como:

$$
\boldsymbol{r}_{1}+\boldsymbol{r}_{4}=\boldsymbol{r}_{2}+\boldsymbol{r}_{3}
$$

Aplicando notación polar a cada término de (4), se obtiene:

$$
r_{1} e^{j \theta_{1}}+r_{4} e^{j \theta_{4}}=r_{2} e^{j \theta_{2}}+r_{3} e^{j \theta_{3}}
$$

Utilizando la ecuación de Euler en (5) y separando la parte real e imaginaria:

$$
\begin{aligned}
& r_{1} \cos \theta_{1}+r_{4} \cos \theta_{4}=r_{2} \cos \theta_{2}+r_{3} \cos \theta_{3} \\
& r_{1} \sin \theta_{1}+r_{4} \sin \theta_{4}=r_{2} \sin \theta_{2}+r_{3} \sin \theta_{3}
\end{aligned}
$$

Para obtener la posición angular $\theta_{3}$, el lado izquierdo del sistema de ecuaciones (6) se expresa en términos de $\theta_{4}$ :

$$
\begin{aligned}
& r_{4} \cos \theta_{4}=r_{2} \cos \theta_{2}+r_{3} \cos \theta_{3}-r_{1} \cos \theta_{1} \\
& r_{4} \sin \theta_{4}=r_{2} \sin \theta_{2}+r_{3} \sin \theta_{3}-r_{1} \sin \theta_{1}
\end{aligned}
$$

Elevando al cuadrado (7) y sumando sus términos, se obtiene la ecuación de Freudenstein en forma compacta [2], la cual se establece como:

$$
A_{1} \cos \theta_{3}+B_{1} \sin \theta_{3}+C_{1}=0
$$

donde:

$$
\begin{aligned}
& A_{1}=2 r_{3}\left(r_{2} \cos \theta_{2}-r_{1} \cos \theta_{1}\right) \\
& B_{1}=2 r_{3}\left(r_{2} \sin \theta_{2}-r_{1} \sin \theta_{1}\right) \\
& C_{1}=r_{1}^{2}+r_{2}^{2}+r_{3}^{2}-r_{4}^{2}-2 r_{1} r_{2} \cos \left(\theta_{1}-\theta_{2}\right)
\end{aligned}
$$


Síntesis óptima de un mecanismo plano para seguimiento de trayectoria utilizando evolución ...

El ángulo $\theta_{3}$ puede ser calculado como una función de los parámetros $A_{1}, B_{1}$, $C_{1}$ y $\theta_{2}$. Dicha solución puede ser obtenida al expresar $\sin \theta_{3}$ y $\cos \theta_{3}$ en términos de $\tan \left(\frac{\theta_{3}}{2}\right)$ como sigue:

$$
\sin \theta_{3}=\frac{2 \tan \left(\frac{\theta_{3}}{2}\right)}{1+\tan ^{2}\left(\frac{\theta_{3}}{2}\right)}, \cos \theta_{3}=\frac{1-\tan ^{2}\left(\frac{\theta_{3}}{2}\right)}{1+\tan ^{2}\left(\frac{\theta_{3}}{2}\right)}
$$

sustituyendo éstas en (8), se obtiene una ecuación lineal de segundo orden:

$$
\left[C_{1}-A_{1}\right] \tan ^{2}\left(\frac{\theta_{3}}{2}\right)+\left[2 B_{1}\right] \tan \left(\frac{\theta_{3}}{2}\right)+A_{1}+C_{1}=0
$$

Resolviendo (13), la posición angular $\theta_{3}$ esta dada por (14).

$$
\theta_{3}=2 \arctan \left[\frac{-B_{1} \pm \sqrt{B_{1}^{2}+A_{1}^{2}-C_{1}^{2}}}{C_{1}-A_{1}}\right]
$$

Un procedimiento similar al anterior se debe llevar a cabo para obtener $\theta_{4}$. A partir de (6) se obtiene la ecuación de Freudenstein que esta dada en forma compacta por:

$$
D_{1} \cos \theta_{4}+E_{1} \sin \theta_{4}+F_{1}=0
$$

donde:

$$
\begin{aligned}
D_{1} & =2 r_{4}\left(r_{1} \cos \theta_{1}-r_{2} \cos \theta_{2}\right) \\
E_{1} & =2 r_{4}\left(r_{1} \sin \theta_{1}-r_{2} \sin \theta_{2}\right) \\
F_{1} & =r_{1}^{2}+r_{2}^{2}+r_{4}^{2}-r_{3}^{2}-2 r_{1} r_{2} \cos \left(\theta_{1}-\theta_{2}\right)
\end{aligned}
$$

Por lo tanto, la posición angualr $\theta_{4}$ esta dada por (19).

$$
\theta_{4}=2 \arctan \left[\frac{-E_{1} \pm \sqrt{D_{1}^{2}+E_{1}^{2}-F_{1}^{2}}}{F_{1}-D_{1}}\right]
$$

En las ecuaciones (14) y (19) se debe elegir el signo apropiado del radical de acuerdo al tipo de configuración del mecanismo de cuatro barras. La Tabla 1 muestra los signos de los radicales de acuerdo a la configuración del mecanismo.

Tabla 1. Selección del signo del radical de acuerdo al tipo de mecanismo

\begin{tabular}{|c|c|c|}
\hline Configuración del mecanismo de cuatro barras & $\theta_{3}$ & $\theta_{4}$ \\
\hline abierta & $+\sqrt{ }$ & $-\sqrt{ }$ \\
\hline cruzada & $-\sqrt{ }$ & $+\sqrt{ }$ \\
\hline
\end{tabular}




\subsection{Cinemática del acoplador}

El punto de interés en el acoplador del mecanismo es $C$, para determinar su posición se tiene que establecer en el sistema $0 X_{r} Y_{r}$ que:

$$
\begin{aligned}
& C_{x r}=r_{2} \cos \theta_{2}+r_{c x} \cos \theta_{3}-r_{c y} \sin \theta_{3} \\
& C_{y r}=r_{2} \sin \theta_{2}+r_{c x} \sin \theta_{3}+r_{c y} \cos \theta_{3}
\end{aligned}
$$

Desde el sistema de coordenadas global, dicho punto se expresa como sigue:

$$
\left[\begin{array}{l}
C_{x} \\
C_{y}
\end{array}\right]=\left[\begin{array}{cc}
\cos \theta_{0} & -\sin \theta_{0} \\
\sin \theta_{0} & \cos \theta_{0}
\end{array}\right]\left[\begin{array}{l}
C_{x r} \\
C_{y r}
\end{array}\right]+\left[\begin{array}{l}
x_{0} \\
y_{0}
\end{array}\right]
$$

Es importante hacer notar que las ecuaciones (20), (21) y las ecuaciones de la cinemática del mecanismo son suficientes para obtener la posición del punto $C$ a lo largo de toda la trayectoria del mecanismo.

\section{Estrategias de optimización}

Una vez que la cinemática del mecanismo se ha establecido apropiadamente, el problema de diseño se debe definir como un problema de optimización numérica, por lo que se deben especificar las relaciones matemáticas que permitan evaluar el desempeño del sistema.

\subsection{Función objetivo}

Como se ha mencionado previamente, en este trabajo se desea determinar el valor de las longitudes de las barras del mecanismo, el ángulo de rotación del sistema de referencia, la distancia entre sistemas de referencia y el conjunto de ángulos del eslabón de entrada que permiten alcanzar los $N$ puntos de precisión.

En el sistema global de coordenadas $0 X Y$, el i-esimo punto de precisión se indica como:

$$
C_{d}^{i}=\left[C_{x d}^{i}, C_{y d}^{i}\right]^{T}
$$

Por otro lado, el conjunto de $N$ puntos de precisión se define como:

$$
\Omega=\left\{C_{d}^{i} \mid i \in N\right\}
$$

Entonces, dado un conjunto de valores de las barras del mecanismo y sus parámetros $x_{0}, y_{0}, \theta_{0}$, cada punto del acoplador se puede expresar como una función de la posición de la barra de entrada.

$$
C^{i}=\left[C_{x}\left(\theta_{2}^{i}\right), C_{y}\left(\theta_{2}^{i}\right)\right]^{T}
$$

Por lo tanto, se desea minimizar la distancia entre los puntos de precisión $C_{d}^{i}$ y los puntos $C^{i}$ que alcanza el mecanismo en la configuración calculada. 
Para cuantificar dicha distancia en todos los puntos de precisión, se propone la siguiente función:

$$
f\left(\theta_{2}^{i}\right)=\sum_{i=1}^{N}\left[\left(C_{x d}^{i}-C_{x}^{i}\right)^{2}+\left(C_{y d}^{i}-C_{y}^{i}\right)^{2}\right]
$$

\subsection{Restricciones de diseño}

Uno de los aspectos más importantes en el diseño del mecanismo es cumplir con el conjunto de restricciones que se imponen a su funcionamiento, las cuales están relacionadas con criterios de movilidad y dimensiones del mismo.

Ley de Grashof. Una de las consideraciones de mayor importancia cuando se diseña un mecanismo es la ley de Grashof, que establece los criterios para los cuales un mecanismo plano de cuatro barras puede asegurar movilidad completa de al menos una de sus barras. La ley de Grashof afirma que para un eslabonamiento plano de cuatro barras, la suma de las longitudes más corta y más larga de los eslabones no puede ser mayor que la suma de las longitudes de los dos eslabones restantes, si se desea que exista una rotación relativa continua entre dos elementos [2]. Si denotamos $s$ a la longitud del eslabón más corto, $l$ a la del más largo y finalmente $p$ y $q$ a las longitudes de los eslabones restantes, la ley de Grashof se establece como:

$$
l+s \leq p+q
$$

En el presente trabajo, la ley de Grashof está dada por:

$$
r_{1}+r_{2} \leq r_{3}+r_{4}
$$

Adicionalmente, con el objetivo de asegurar que el método de solución produce mecanismos que cumplen con la ley de Grashof, se establecen la siguientes restricciones:

$$
\begin{aligned}
r_{2} & <r_{3} \\
r_{3} & <r_{4} \\
r_{4} & <r_{1}
\end{aligned}
$$

Secuencia de ángulos de entrada. Debido a que el problema de síntesis que se aborda en el presente trabajo es el de generación de trayectoria sin sincronización prescrita, es necesario asegurar que los valores de los ángulos de la manivela sean ordenados en forma ascendente o descendente. Si denotamos el valor del ángulo de la manivela en el $i$-esimo punto de precisión como $\theta_{2}^{i}$, entonces se debe cumplir que:

$$
\theta_{2}^{1}<\theta_{2}^{2}<\ldots<\theta_{2}^{N}
$$

donde $N$ es el número de puntos de precisión. 


\section{Diseño óptimo del mecanismo}

Para la obtención del conjunto óptimo de los parámetros de diseño se debe parametrizar el sistema de acuerdo a las variables involucradas en el mismo. Una descripción apropiada de variables permite al diseñador una amplia posibilidad de reconfiguración del sistema.

\subsection{Variables de diseño}

Sea el vector de variables de diseño para el mecanismo de cuatro barras, establecido como:

$$
\begin{aligned}
\boldsymbol{p} & =\left[p_{1}, p_{2}, p_{3}, p_{4}, p_{5}, p_{6}, p_{7}, p_{8}, p_{9}, p_{10}, p_{11}, p_{12}, p_{13}, p_{14}, p_{15}\right]^{T} \\
& =\left[r_{1}, r_{2}, r_{3}, r_{4}, r_{c x}, r_{c y}, \theta_{0}, x_{0}, y_{0}, \theta_{2}^{1}, \theta_{2}^{2}, \theta_{2}^{3}, \theta_{2}^{4}, \theta_{2}^{5}, \theta_{2}^{6}\right]^{T}
\end{aligned}
$$

donde las primeras cuatro variables corresponden a las longitudes de la barras del mecanismo, las siguientes dos al punto $C$ del acoplador, las tres subsecuentes a la orientacion entre sistemas coordenados de referencia y las últimas seis a la secuencia de valores del ángulo de la barra de entrada del mecanismo.

\subsection{Problema de optimización}

Sea el problema de optimización numérica mono objetivo descrito por (34) hasta (47), para obtener la solución al problema de diseño de síntesis para generación de trayectoria de un mecanismo de cuatro barras:

$$
\begin{aligned}
& \operatorname{Min} f(\boldsymbol{p})=\sum_{i=1}^{N}\left[\left(C_{x d}^{i}-C_{x}^{i}\right)^{2}+\left(C_{y d}^{i}-C_{y}^{i}\right)^{2}\right] \\
& \boldsymbol{p} \in \mathbb{R}^{15}
\end{aligned}
$$

sujeto a:

$$
\begin{aligned}
& g_{1}(\boldsymbol{p})=p_{1}+p_{2}-p_{3}-p_{4} \leq 0 \\
& g_{2}(\boldsymbol{p})=p_{2}-p_{3} \leq 0 \\
& g_{3}(\boldsymbol{p})=p_{3}-p_{4} \leq 0 \\
& g_{4}(\boldsymbol{p})=p_{4}-p_{1} \leq 0 \\
& g_{5}(\boldsymbol{p})=p_{10}-p_{11} \leq 0 \\
& g_{6}(\boldsymbol{p})=p_{11}-p_{12} \leq 0 \\
& g_{7}(\boldsymbol{p})=p_{12}-p_{13} \leq 0 \\
& g_{8}(\boldsymbol{p})=p_{13}-p_{14} \leq 0 \\
& g_{9}(\boldsymbol{p})=p_{14}-p_{15} \leq 0
\end{aligned}
$$


con las cotas:

$$
\begin{array}{rlrl}
0 & \leq p_{i} \leq 60, & i=1,2,3,4 \\
-60 & \leq p_{i} \leq 60, \quad i=5,6,8,9 \\
0 & \leq p_{i} \leq 2 \pi, \quad i=7,10,11,12,13,14,15
\end{array}
$$

y los puntos de precisión:

$$
\Omega=\{(20,20),(20,25),(20,30),(20,35),(20,40),(20,45)\}
$$

\section{Algoritmo de optimización}

El algoritmo de Evolución Diferencial (DE) es uno de los paradigmas mas populares dentro de la computación evolutiva, ya que resuelve de manera eficiente problemas no lineales, no diferenciables y multimodales [11]. DE parte de una población inicial de soluciones candidatas arbitrarias, y en cada generación se producen individuos de prueba aplicando los operadores de reproducción (cruza y mutación). La aptitud de cada individuo nuevo se evalúa para que compita con el individuo padre, y así determinar cuál de ellos se conservará para la generación siguiente. Una de las principales ventajas de la evolución diferencial es su número reducido de parámetros de control. Solamente se requieren tres parámetros de entrada para controlar el proceso de búsqueda; esto es, el tamaño de la población o conjunto de soluciones $N$, la constante de diferenciación $F$ que controla la amplificación de la variación diferencial y el parámetro de control de cruza $C R$ [3]. Las características generales de esta técnica son:

- Representación de individuos

- Selección de padres

- Recombinación o cruza

- Mutación

- Selección de sobrevivientes y variantes.

El pseudocódigo correspondiente a la evolución diferencial se muestra en el Algoritmo 5.1: 


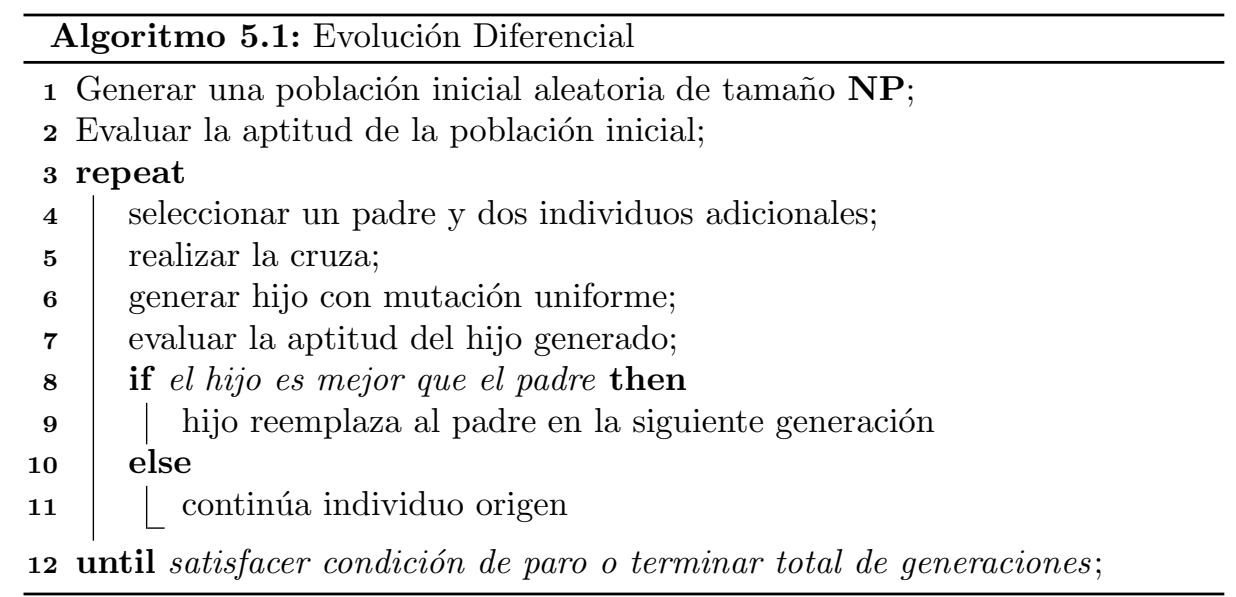

En la etapa de competencia para sustitución generacional se utilizaron las reglas de factibilidad de Deb [8]:

1. Entre dos individuos factibles, se escoge al que tenga la mejor función objetivo.

2. Entre un individuo factible y otro no factible, se escoge al factible.

3. Entre dos individuos no factibles, se escoge al que tenga un valor menor en la suma de violaciones a las restricciones.

\subsection{Implementación computacional}

La implementación del algoritmo se programó en MATLAB R2013a, y las corridas se llevaron a cabo en una plataforma computacional con las siguientes características: procesador Intel Core i7 @ $1.75 \mathrm{GHz}$, con 8Gb de memoria RAM y sistema operativo Windows 8.

En el programa se utilizan dos funciones, secuencia y error_puntero. En secuencia se evalúan las restricciones en el orden de los ángulos $\theta_{2}$ correspondientes a los puntos a seguir por el mecanismo; el incumplimiento de estas restricciones se convierte en un solo valor, cuya magnitud indica el grado de violación de la secuencia. Por su parte, en error_puntero se observa que el individuo sea factible calculando la suma de las violaciones a las restricciones derivadas de la Ley de Grashof; si el vector de variables propuesto es factible se calcula la funcion objetivo, en caso contrario se le asigna un valor muy grande, F. $\mathrm{O}=1000$. Existe una situación especial en la que el individuo es factible pero produce un mecanismo de doble balancín, en cuyo caso se penaliza la funcion objetivo.

\section{Resultados}

Se llevó a cabo un conjunto de cincuenta corridas del algoritmo propuesto, de las cuales se seleccionaron las diez con mejores resultados de la función objetivo. Inicialmente, se realizó la calibración de los parámetros del algoritmo 
Síntesis óptima de un mecanismo plano para seguimiento de trayectoria utilizando evolución ...

buscando una mayor convergencia del resultado dentro de la zona factible. La puesta a punto se obtuvo con el siguiente conjunto de parámetros: tamaño de la población $\mathrm{NP}=100$, número máximo de generaciones $\mathrm{GMAX}=5,000$, factor de escalamiento $\mathrm{F}=[0.8,1]$, y factor de cruza $\mathrm{CR}=[0.3,0.9]$; estos dos últimos valores se generaron en forma aleatoria dentro de los rangos especificados.

Las Tablas 2 y 3 muestran los valores de los vectores solución obtenidos en las mejores ejecuciones. Como puede observarse, todos los valores caen dentro de los límites marcados por las restricciones de diseño; por cuestiones de espacio sólo se muestran los primeros tres dígitos decimales, aunque en la corrida de las simulaciones se generaron datos con una precisión de catorce posiciones decimales. La Tabla 4 muestra la magnitud del error obtenido por el algoritmo, con un excelente valor promedio del orden de $10^{-14}$ e incluso alcanzando el nivel de $10^{-29}$ para la mejor corrida. Finalmente, en la Tabla 5 se pueden observar los tiempos de ejecución correspondientes, los cuales son relativamente cortos, en el orden de $2.6 \mathrm{~min}$.

Tabla 2. Mejores diez vectores de solución.

\begin{tabular}{|l|l|l|l|l|l|l|l|l|}
\hline $\mathbf{N}$ & $r_{1}$ & $r_{2}$ & $r_{3}$ & $r_{4}$ & $r_{c x}$ & $r_{c y}$ & $\theta_{0}$ & $x_{0}$ \\
\hline 1 & 38.463 & 8.544 & 27.892 & 37.388 & 36.897 & 18.156 & 3.943 & -9.429 \\
\hline 2 & 38.045 & 8.517 & 27.934 & 38.038 & 37.631 & 16.453 & 3.952 & -9.323 \\
\hline 3 & 38.003 & 8.384 & 28.182 & 36.679 & 37.614 & 18.679 & 3.965 & -10.314 \\
\hline 4 & 30.642 & 7.877 & 28.909 & 30.619 & 47.306 & 19.146 & 4.253 & -21.061 \\
\hline 5 & 37.415 & 8.538 & 27.102 & 37.227 & 36.230 & 16.332 & 3.940 & -8.2637 \\
\hline 6 & 38.039 & 8.394 & 28.628 & 37.501 & 38.875 & 17.736 & 3.978 & -10.915 \\
\hline 7 & 35.483 & 8.052 & 28.653 & 34.413 & 40.594 & 19.211 & 4.056 & -13.694 \\
\hline 8 & 37.568 & 8.338 & 28.556 & 36.949 & 38.986 & 17.899 & 3.988 & -11.191 \\
\hline 9 & 32.332 & 7.730 & 27.220 & 31.033 & 39.811 & 19.179 & 4.108 & -13.829 \\
\hline 10 & 36.624 & 8.184 & 28.110 & 35.295 & 38.323 & 18.826 & 4.001 & -11.270 \\
\hline
\end{tabular}

Con respecto al análisis de convergencia en las corridas seleccionadas, en la Figura 2 se aprecia que el valor de la función objetivo tiende a converger aproximadamente después de las primeras cincuenta generaciones, mientras que en la Figura 3 se observa que la totalidad de las soluciones (individuos) entran a la zona factible en ese mismo lapso. Ambas gráficas se ajustan al comportamiento general de los algoritmos evolutivos [9] y demuestran la capacidad de la evolución diferencial para localizar el mínimo global en un tiempo corto.

\section{Conclusiones}

En este articulo se presentó una técnica para la síntesis de un mecanismo de cuatro barras para seguimiento de trayectorias, con base en el algoritmo de Evolución Diferencial. Se realizó una modificación al algoritmo original para aplicar los criterios de factibilidad de Deb en la etapa de concurso entre los 
Eduardo Vega-Alvarado, Eric Santiago-Valentín, Alvaro Sánchez-Márquez, et al.

Tabla 3. Mejores vectores de solución (cont.) y función objetivo correspondiente.

\begin{tabular}{|l|l|l|l|l|l|l|l|}
\hline$y_{0}$ & $\theta_{2}{ }^{1}$ & $\theta_{2}{ }^{2}$ & $\theta_{2}{ }^{3}$ & $\theta_{2}{ }^{4}$ & $\theta_{2}{ }^{5}$ & $\theta_{2}{ }^{6}$ & F.O. \\
\hline 59.705 & 1.625 & 2.421 & 2.936 & 3.429 & 3.965 & 5.325 & $5.048 \mathrm{E}-29$ \\
\hline 59.223 & 1.750 & 2.465 & 2.976 & 3.472 & 4.021 & 5.126 & $5.048 \mathrm{E}-29$ \\
\hline 59.911 & 1.625 & 2.422 & 2.940 & 3.436 & 3.977 & 5.227 & $6.310 \mathrm{E}-29$ \\
\hline 59.641 & 1.912 & 2.481 & 2.946 & 3.396 & 3.869 & 4.465 & $7.573 \mathrm{E}-29$ \\
\hline 58.625 & 1.689 & 2.442 & 2.958 & 3.457 & 4.004 & 5.262 & $1.161 \mathrm{E}-27$ \\
\hline 59.960 & 1.741 & 2.459 & 2.969 & 3.464 & 4.009 & 5.048 & $5.452 \mathrm{E}-27$ \\
\hline 59.989 & 1.735 & 2.445 & 2.956 & 3.448 & 3.984 & 4.873 & $2.930 \mathrm{E}-22$ \\
\hline 59.894 & 1.735 & 2.455 & 2.967 & 3.462 & 4.007 & 5.028 & $8.707 \mathrm{E}-18$ \\
\hline 58.658 & 1.673 & 2.414 & 2.936 & 3.435 & 3.976 & 4.848 & $5.161 \mathrm{E}-17$ \\
\hline 59.697 & 1.646 & 2.424 & 2.945 & 3.445 & 3.991 & 5.092 & $1.774 \mathrm{E}-13$ \\
\hline
\end{tabular}

Tabla 4. Estadísticas de las simulaciones numéricas.

\begin{tabular}{|c|c|}
\hline Mejor & $5.04870 \mathrm{E}-29$ \\
\hline Promedio & $1.77509 \mathrm{E}-14$ \\
\hline Peor & $1.77449 \mathrm{E}-13$ \\
\hline Desviación estándar & $5.32328 \mathrm{E}-14$ \\
\hline
\end{tabular}

Tabla 5. Tiempo de ejecución de las simulaciones numéricas.

\begin{tabular}{|c|c|c|c|c|c|c|c|c|c|c|}
\hline Simulación & 1 & 2 & 3 & 4 & 5 & 6 & 7 & 8 & 9 & 10 \\
\hline Tiempo (min) & 2.63 & 2.61 & 2.76 & 2.63 & 2.63 & 2.60 & 2.65 & 2.65 & 2.63 & 2.58 \\
\hline
\end{tabular}

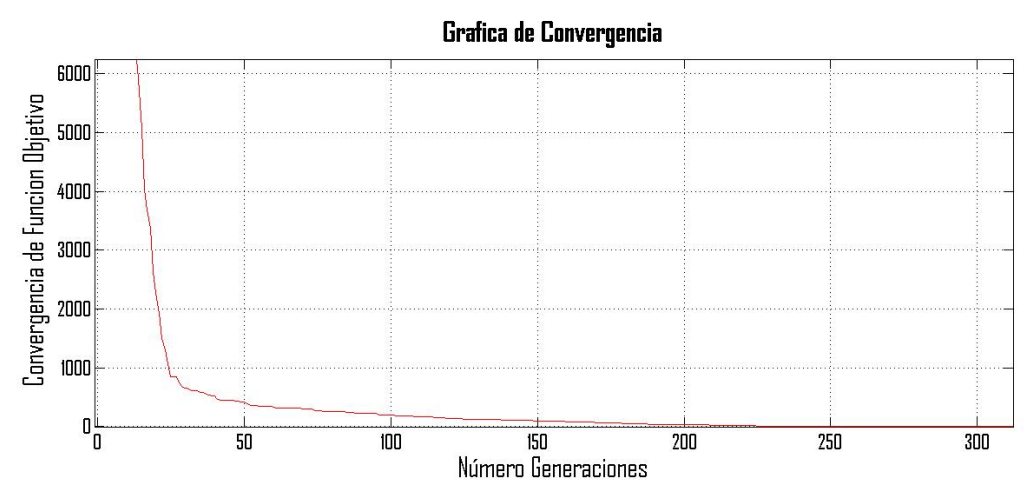

Fig. 2. Convergencia de la función objetivo hacia el valor óptimo.

individuos originales y los de nueva creación, que produjo una mejora en la toma de decisión para conformar la generación siguiente. De los resultados obtenidos, se pudo establecer que este método de solución para la optimización de mecanismos produce buenos resultados desde el punto de vista del diseño en 


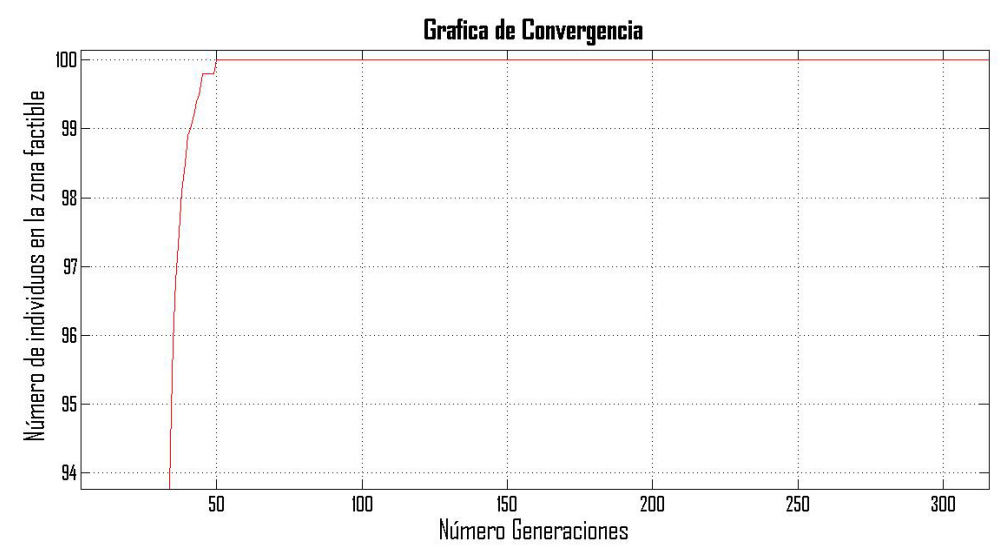

Fig. 3. Promedio de individuos factibles por generación, considerando 5000 generaciones y 100 individuos.

ingenieria, sin requerir el uso de recursos de cómputo extensivos y con un tiempo de respuesta razonable. Si bien el algoritmo aquí propuesto fue desarrollado para el caso específico de un mecanismo de cuatro barras, su implementación simple permite que pueda utilizarse para diseñar otro tipo de mecanismos. En este sentido, la dificultad mayor radica en la correcta interpretación y formulación de las restricciones del problema específico. Así mismo, la sintonización de los parámetros del algoritmo requiere atención especial; la generación aleatoria de los mismos produce buenos resultados, aunque en algunos casos es necesario un ajuste más preciso.

Agradecimientos. El primer autor es el autor para correspondencia. Todos los autores agradecen el apoyo del Instituto Politécnico Nacional a través de la SIP vía el proyecto SIP-20141257. El segundo autor agradece al CONACyT por la beca para estudios de posgrado en el CIDETEC-IPN.

\section{Referencias}

1. Norton, R.L.: Diseño de Maquinaria, una Introducción a la Síntesis y al Análisis de Mecanismos y Máquinas, McGraw Hill, México (1995)

2. Shigley, J.E., Uicker, J.J. Jr.: Teoría de Máquinas y Mecanismos, McGraw Hill, México (1988)

3. Boussaid, I., Lepagnot, J., Siarry, P.: A Survey on Optimization Metaheuristics. Information Sciences. 237, 82-117 (2013)

4. Cabrera, J.A., Simon, A., Prado, A.: Optimal Synthesis of Mechanisms with Genetic Algorithms. Mechanism and Machine Theory. 37, 1165-1177 (2002)

5. Bulatović, R.R., Dordević, S.R.: On the Optimum Synthesis of a Four-Bar Linkage Using Differential Evolution and Method of Variable Controlled Deviations. Mechanism and Machine Theory. 44, 235-246 (2009) 
Eduardo Vega-Alvarado, Eric Santiago-Valentín, Alvaro Sánchez-Márquez, et al.

6. Acharyya, S.K., Mandal, M.: Performance of EAs for Four-Bar Linkage Synthesis. Mechanism and Machine Theory. 44, 1784-1794 (2009)

7. Matekar, S.B., Gogate, G.R.,: Optimum Synthesis of Path Generating Four-Bar Mechanisms Using Differential Evolution and a Modified Error Function. Mechanism and Machine Theory. 52, 158-179 (2012)

8. Deb, K.: An Efficient Constraint Handling Method for Genetic Algorithms. Computer Methods in Applied Mechanics and Engineering. 186, 311-338 (2000)

9. Eiben, A.E., Smith, J.E.: Introduction to Evolutionary Computing, Springer Verlag, New York (2007)

10. Pérez Moreno, R.: Análisis de Mecanismos y Problemas Resueltos, Alfaomega Grupo Editor S.A. de C.V., México (2006)

11. Price, K.V.: An Introduction to Differential Evolution, New Ideas in Optimization, Mc Graw Hill, UK (1999) 